\title{
RESEARCH HIGHLIGHT Unconventional secretion: cargo channeling by TMED10
}

\author{
Tan A. Nguyen ${ }^{1}$ and Jayanta Debnath (D) ${ }^{1}$ \\ Cell Research (2020) 30:713-714; https://doi.org/10.1038/s41422-020-0382-x
}

\begin{abstract}
Cytosolic proteins lacking a signal peptide are often loaded into vesicles and secreted via unconventional protein secretion pathways. A recent paper in Cell identifies the transmembrane protein TMED10 as a protein channel for the vesicular translocation and secretion of mature IL-1 $\beta$ and other leaderless cargoes.
\end{abstract}

Traditionally, protein secretion in eukaryotes involves the recognition of a signal peptide and the translocation and export of the protein through the ER-Golgi via the translocon SEC61 and COPII and COPI vesicles. Alternatively, numerous cytosolic proteins lacking a signal peptide, termed leaderless proteins, are secreted into the extracellular milieu through a diverse cellular process, collectively termed unconventional protein secretion (UPS). Leaderless proteins can directly translocate across the plasma membrane via pores (type I unconventional secretion) or ATP-binding cassette (ABC) transporters (type II); alternatively, they are loaded and trafficked through vesicular compartments which are delivered to the plasma membrane (type III). ${ }^{1}$ Precisely understanding how these leaderless cargoes are loaded into vesicular intermediates remains a fundamental question in the field. Zhang and colleagues now provide a groundbreaking insight into vesicle-mediated UPS via the transmembrane channel protein, TMED-10, in a process termed TMED10-channeled UPS (THU). ${ }^{2}$

A highly investigated protein secreted via UPS pathways is the pro-inflammatory cytokine IL-1 $\beta$, which is catalytically processed to a mature form (mlL-1 $\beta$ ) and released upon specific stimuli such as inflammasome activation and pyroptosis. ${ }^{3}$ Employing an elegant mass spectrometry approach, the authors identify 11 candidate transmembrane proteins that interact with $\mathrm{mlL}-1 \beta$ during its translocation into vesicular carriers. ${ }^{2}$ Subsequent loss-offunction experiments reveal that loss of TMED10 impairs secretion of $\mathrm{mL}-1 \beta$ in macrophage and neutrophil cell lines. Importantly, TMED10 does not impact secretion of IL- $1 \beta$ through gasdermin D (GSDMD), suggesting that THU is distinct from and independent of the previously described GSDMD pore-forming secretion pathway. ${ }^{4}$ However, the relative physiological contribution of GSDMD versus TMED10-mediated IL- $\beta$ release remains unclear. Nevertheless, conditional deletion of TMED10 in myeloid cells of mice results in reduced serum IL-1 $\beta$ and prolonged survival in a model of cecal ligation and puncture (CLP)-induced septic shock. An important follow-up will be to determine whether inhibiting IL-1 $\beta$ secretion via THU has therapeutic potential for the treatment of various human inflammatory diseases.

TMED10 contains a single transmembrane domain, a luminal signal peptide (SS), a Golgi dynamics (GOLD) domain, a coil-coil (CC) domain and a C-terminal tail (CT) facing the cytoplasm. Using a series of truncation mutants, the authors uncover that the $C T$ domain of TMED10 directly interacts with and is required for the secretion of $\mathrm{mIL}-1 \beta$ and other known UPS cargoes. Interestingly, two proteins previously shown to be secreted via UPS in a TMED10-dependent manner, $\mathrm{Tau}^{5}$ and Annexin $\mathrm{A} 1{ }^{6}$ do not interact with TMED-CT, raising the possibility that TMED10 may indirectly regulate the secretion of such UPS targets. Consistent with this notion, the authors identify a putative TMED10 recognition motif within $\mathrm{mL}-1 \beta$ termed 'motif 1 '. Mutation of this motif results in reduced secretion of $\mathrm{mL}-1 \beta$, while fusion of this TMED10 recognition motif to an mCherry reporter is sufficient to promote interaction with TMED10 and the subsequent secretion of mCherry via THU. Remarkably, the authors note that the secretion of other leaderless cargoes, such as $\mathrm{HMGB}^{7}$ and asynuclein, ${ }^{8}$ are not regulated by THU. Future mutational and functional studies will undoubtedly shed further light on the conservation of this identified motif across other known UPS cargoes and potentially lead to therapeutic strategies to modulate the unconventional secretion of pro-inflammatory cytokines or pathogenic protein aggregates.

In previous work, the authors observed that the vesicular translocation of IL- $1 \beta$ requires an initial protein unfolding step and the association with the chaperone protein HSP90 via the recognition of two KFERQ-like sequences. ${ }^{9}$ This also appears to be the case for TMED10-mediated IL-1 $\beta$ secretion since both predenaturation of $\mathrm{mIL}-1 \beta$ with urea and HSP90A overexpression enhance mIL-1 $\beta$ vesicular entry and secretion. Notably, previous studies implicated a secretory autophagy pathway, ${ }^{7}$ in which $\mathrm{mlL}-1 \beta$ (but not pro-IL-1 $\beta$ ) was incorporated within the intermembrane space of an autophagosome-like vesicular intermediate and subsequently secreted extracellularly. ${ }^{9}$ Despite these parallels, the relationship between THU and secretory autophagy remains unclear. Notably, TMED10 co-localizes with the ER-Golgi intermediate compartment (ERGIC) and mediates entry of $\mathrm{mL}-1 \beta$ and other UPS cargoes including mlL-1a, mIL-36a, mlL-36RA, mIL-37, mlL-38, and HSPB5. Further studies are needed to illuminate whether TMED10-mediated residing of cargoes within the ERGIC can intersect with secretory autophagosomes, multivesicular bodies (MVBs) or the recently described LC3-dependent extracellular vesicle loading and secretion (LDELS) pathway. ${ }^{10}$

Finally, the authors further characterize the mechanism by which TMED10, a single transmembrane protein, is able to translocate diverse proteins of varying sizes. Cross-linking experiments reveal that TMED10 can form higher order oligomeric structures following the production of $\mathrm{mLL}-1 \beta$ in THP-1 cells. Interestingly, this oligomerization is both dependent on and induced by the presence of UPS competent cargo; accordingly, enforced expression of TMED10-dependent cargoes such as $\mathrm{mlL}-1 \beta$, mlL-1a and HSPB5 is sufficient to drive TMED10 oligomerization. Furthermore, the recognition of motif 1 via the CT domain of TMED10 leads to the stabilization of the TMED10 channel pore, which gradually monomerizes following secretion. 


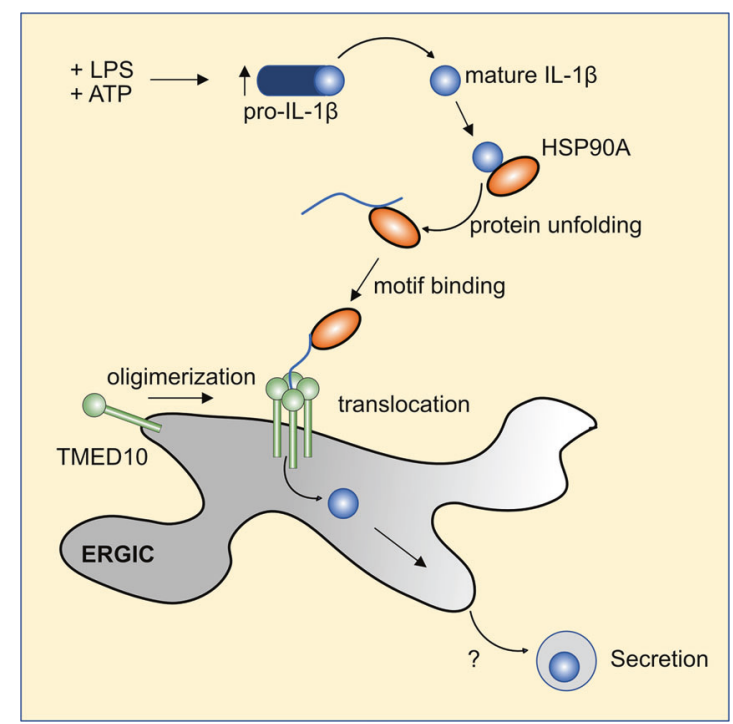

Fig. 1 TMED10-mediated UPS. The cytosolic chaperone HSP90A binds and unfolds protein cargo destined for UPS, such as mature IL-1 $\beta$. This HSP90A-bound cargo interacts with the C-terminal tail of TMED10 via a novel recognition motif, resulting in the oligomerization of TMED10 to form a protein channel that translocates the cargo into the lumen of the ERGIC for subsequent secretion outside the cell via a vesicular intermediate.
Altogether, these results support a model by which UPS cargo, such as mlL-1 $\beta$, is unfolded upon binding to cytosolic chaperone HSP90A. This cargo subsequently interacts with the C-terminal tail of TMED10 via a novel recognition motif that induces the oligomerization of TMED10 to form a protein channel. The TMED10 channel subsequently binds HSP90B1 and translocates the cargo into the lumen of the ERGIC for secretion via a currently undefined mechanism (Fig. 1). This work provides new perspectives into how proteins lacking signal peptides are incorporated into vesicles for secretion via UPS. Given the important role that THU plays in the secretion of mature IL-1 $\beta$, one of the most intensely studied UPS cargoes and known activators of auto-inflammatory diseases, this work will undoubtedly spur further efforts to understand and harness UPS to treat a wide array of human diseases such as autoimmune disease, neurodegeneration and cancer.

\section{REFERENCES}

1. Kim, J., Gee, H. Y. \& Lee, M. G. J. Cell Sci. 131, jcs213686 (2018).

2. Zhang, M. et al. Cell 181, 637-652 (2020).

3. Monteleone, M., Stow, J. L. \& Schroder, K. Cytokine 74, 213-218 (2015).

4. Evavold, C. L. et al. Immunity 48, 35-44 (2018).

5. Merezhko, M. et al. Cell Rep. 25, 2027-2035 (2018).

6. Popa, S. J., Stewart, S. E. \& Moreau, K. Semin. Cell Dev. Biol. 83, 42-50 (2018).

7. Dupont, N. et al. EMBO J. 30, 4701-4711 (2011).

8. Jang, A. et al. J. Neurochem. 113, 1263-1274 (2010).

9. Zhang, M., Kenny, S. J., Ge, L., Xu, K. \& Schekman, R. Elife 4, e11205 (2015).

10. Leidal, A. M. et al. Nat. Cell Biol. 22, 187-199 (2020) 